\title{
USP39 regulates the growth of SMMC-7721 cells via FoxM1
}

\author{
XIANWEN YUAN ${ }^{1,2^{*}}$, XITAI SUN ${ }^{1,3^{*}}$, XIAOLEI SHI ${ }^{1,2}$, CHUNPING JIANG $^{1,2}$, \\ DECAI YU ${ }^{1,2}$, WEIWEI ZHANG ${ }^{1,2}$ and YITAO DING ${ }^{1,2}$ \\ ${ }^{1}$ Department of Hepatobiliary Surgery, The Affiliated Drum Tower Hospital of Nanjing University Medical School;
${ }^{2}$ Institute of Hepatobiliary Surgery, Nanjing University; ${ }^{3}$ Center of Minimally Invasive Surgery,
The Affiliated Drum Tower Hospital of Nanjing University Medical School, Nanjing, Jiangsu 210008, P.R. China
}

Received November 13, 2015; Accepted December 1, 2016

DOI: $10.3892 / \mathrm{etm} .2017 .4115$

\begin{abstract}
The present study investigated ubiquitin specific peptidase 39 (USP39) gene knockdown on SMMC-7721 cells in vitro and in vivo, and the role of USP39 in regulating the growth of hepatocellular carcinoma (HCC). Two small interfering RNAs (siRNA) were constructed, which targeted the USP39 gene and control sequences were synthesized and inserted into a pGCSIL-GFP lentiviral vector. The full length of USP39 cDNA was amplified by polymerase chain reaction (PCR) and cloned into pEGFP-N2, and the recombinant plasmids were transfected into cells. Knockdown efficiency and upregulation of USP39 was determined by reverse transcription-quantitative PCR and western blotting. The impact of USP39 on the growth of SMMC-7721 cells in vitro was examined using an MTT assay, colony formation, flow cytometry (FCM) and immunohistochemical staining. The impact of USP39 on the growth of SMMC-7721 cells in vivo was examined by assessing tumorigenicity in nude mice. Western blotting was performed to examine the mechanism of USP39 regulation on SMMC-7721 cell growth. Recombinant vectors containing specific and scrambled USP39 siRNA sequences were constructed and transfected into SMMC-7721 cells. USP39 knockdown inhibited cell proliferation and colony formation in SMMC-7721 cells, while upregulation of USP39 promoted the growth of tumor cells. FCM indicated that USP39 knockdown led to G2/M arrest and induced apoptosis in SMMC-7721 cells. USP39 knockdown inhibited xenograft tumor growth in nude mice and led to the downregulation of the transcription factor Forkhead Box M1 (FoxM1). Gene expression of FoxM1 targets, including polo-like kinase 1,
\end{abstract}

Correspondence to: Professor Yitao Ding, Department of Hepatobiliary Surgery, The Affiliated Drum Tower Hospital of Nanjing University Medical School, 321 Zhongshan Road, Nanjing, Jiangsu 210008, P.R. China

E-mail: dytnanjing1983@126.com

*Contributed equally

Key words: hepatocellular carcinoma, USP39, G2/M arrest, Forkhead Box M1 cyclin B1 and centromere protein A also decreased following USP39 knockdown. The results suggest that knockdown of USP39 inhibits the growth of HCC in vitro and in vivo, potentially through the induction of $\mathrm{G} 2 / \mathrm{M}$ arrest by regulating the pre-mRNA splicing of FoxM1.

\section{Introduction}

Hepatocellular carcinoma (HCC) is among the most prevalent forms of cancer and is the third most common cause of cancer-associated mortality worldwide, resulting in $\sim 600,000$ mortalities per year $(1,2)$. Inhibitors that target their corresponding pathways in HCC have achieved promising results (3), however the detailed molecular mechanisms of HCC tumorigenesis remain unknown.

During cell cycle progression, signaling pathways monitor the upstream events that occur prior to proceeding to the next phase. These regulatory switches are known as cell cycle checkpoints (4). Forkhead Box M1 (FoxM1) is a mammalian transcription factor that regulates mitotic entry and subsequent execution of the mitotic program by controlling the expression of a cluster of G2/M target genes (5). The human FOXM1 gene consists of 10 exons, including exon Va and exon VIIa that are alternatively spliced. These splices give rise to three distinct isoforms of FOXM1:FOXM1a, FOXM1b, and FOXM1c (6-8). FOXM1a harbors exon Va and exon VIIa and is transcriptionally inactive owing to disruption of its transactivation domain (TAD) by exon VIIa. FOXM1b contains neither of these two exons and FOXM1c only contains exon Va. These two isoforms are transcriptionally active and activate expression of their target genes via different mechanisms (7). In mice, FoxM1 has been reported to regulate the expression of cell division cycle 25 (Cdc25) B, Aurora B kinase, survivin, polo-like kinase (PLK)1, centromere proteins (CENP) A and $\mathrm{B}$ and cyclin B1 (9).

PLK was first identified in the polo gene of Drosophila melanogaster and functional mutations of PLK cause various defects in mitosis (10). Five mammalian PLK family members have been identified so far: PLK1, 2, 3, 4 and $5(11,12)$. PLK1 is the most investigated member of the family and has been widely pursued as an oncology target $(13,14)$. The PLK1 protein activates $\mathrm{Cdc} 25 \mathrm{c}$ phosphatase, resulting in the removal of inhibitory phosphorylations from the cell cycle regulatory protein cyclin dependent kinase 1 (CDK1)/cyclin B (15). 
A phosphorylated peptide motif has been detected in PLK1 substrates including $\mathrm{Cdc} 25 \mathrm{c}$, which regulates the activation of CDK1/cyclin B, and the crystal structures of peptides based on this motif and in complex with the Polo box domain have been determined $(16,17)$.

Ubiquitin specific peptidase 39 (Usp39) has been identified as a novel factor that maintains the spindle checkpoint and supports successful cytokinesis. It has been demonstrated that USP39 is an important part of the spliceosome, which is composed of the small nuclear ribonucleoproteins (snRNPs) $\mathrm{U} 1, \mathrm{U} 2$, U4, U5 and U6, as well as $>200$ polypeptides $(18,19)$. A specific reduction in Aurora B mRNA levels following the depletion of USP39 has been observed, however, forced expression of Aurora B is not sufficient to reverse the damage to the checkpoints caused by USP39 depletion in U2OS cells (20). In addition, the zebrafish USP39 mutation induces G1/S arrest by a retinoblastoma 1 (rb1) splicing defect and transcription factor e2f4 (e2f4) is the target of USP39 (21). USP39 mutations contribute to the adenohypophyseal sensitivity of rb1 and e2f4 that causes pituitary tumorigenesis (21). USP39 may act as an oncogenic factor in breast cancer since the downregulation of USP39 induces the apoptosis of MCF-7 cells in vitro. It was demonstrated that the inhibition of USP39 induced G0/G1phase arrest and the apoptosis of breast cancer cells (22).

These results suggest that USP39 may be a potential molecular target for cancer therapy. However, the roles of USP39 in HCC are not well studied. The present study aimed to investigate the potential functions of USP39 in HCC. It has been determined that levels of USP39 expression are higher in liver cancer cells, indicating that USP39 may act as a novel target in liver cancer (23). To test this hypothesis, USP39 expression was attenuated by small interfering RNA (siRNA) and USP39 expression was upregulated in the HCC cell line SMMC7721. It was found that knockdown of USP39 decreased cell proliferation in vitro and inhibited tumor growth in vivo, while overexpression of USP39 promoted the growth of HCC. Additionally, it was demonstrated that the inhibition of USP39 suppressed the tumorigenesis of HCC by inhibiting FoxM1 pre-mRNA splicing.

\section{Materials and methods}

Antibodies and cell culture. Antibodies against GAPDH (cat. no. sc-166574), FoxM1 (cat. no. sc-376471), cyclin B1 (cat. no. sc-7393), CENPA (cat. no. sc-22787), PLK1 (cat. no. sc-55504; dilution, 1:1,000 for all) for western blot analysis and against proliferating cell nuclear antigen (PCNA; cat. no. sc-56; dilution, 1:500) for immunohistochemistry (IHC) were purchased from Santa Cruz Biotechnology, Inc. (Dallas, TX, USA). Antibodies against USP39 (cat. no. ab131332; dilution, 1:5,000) were purchased from Abcam (Cambridge, UK).

The human HCC cell line SMMC-7721 was purchased from the Shanghai Institutes for Biological Sciences. Cells were cultured in RPMI-1640 medium (cat. no. 11875) supplemented with $10 \%$ fetal bovine serum (cat. no. 10099; both, Thermo Fisher Scientific, Inc., Waltham, MA, USA), 100 U/ml penicillin and $100 \mu \mathrm{g} / \mathrm{ml}$ streptomycin (complete medium) and maintained at $37^{\circ} \mathrm{C}$ and $5 \% \mathrm{CO}_{2}$.
Transfection. The siRNAs targeting USP39 knockdown (KD): ACCAAGTTGCCTCCATATCTA], (KD\#: CCAGAC AACTATGAGATCATCGATT) and control siRNA negative control (NC): TTCTCCGAACGTGTCACGT) were transformed into short hairpin RNA (shRNA) (stem-loop-stem structure) and cloned into the pGCSIL-GFP lentiviral vector (GeneChem Co. Ltd., Shanghai, China) following AgeI/EcoRI digestion. The recombinant plasmid and two virus packaging plasmids (GeneChem Co., Ltd) were transfected into human $293 \mathrm{~T}$ cells, which were purchased from the Shanghai Institutes for Biological Sciences (Shanghai, China) using Lipofectamine $^{\mathrm{TM}} 3000$ (Invitrogen; Thermo Fisher Scientific, Inc.) following the manufacturer's protocol. After 3 days incubation, at $37^{\circ} \mathrm{C}$ and in an atmosphere containing $5 \%$ $\mathrm{CO}_{2}$, the lentivirus from the complete medium was collected. For stable infection, HCC cell lines were cultured in 6-well plates and infected with USP39 shRNA-expressing lentivirus (USP39-shRNA) or non-silencing shRNA-expressing lentivirus (control) with a multiplicity of infection (MOI) of 10 . Five days after infection, cells were observed using fluorescence microscopy (DMI4000B; Leica Microsystems GmbH, Wetzlar, Germany). For USP39 overexpression, full length USP39 cDNA was amplified by reverse transcription (RT) polymerase chain reaction (PCR). Total RNA was extracted from SMMC-7721 cell lines using an RNAsimple Total RNA kit (Tiangen Biotech Co., Ltd., Beijing, China). Total RNA was converted into cDNA using an RT kit (Takara Biotechnology Co., Ltd., Dalian, China), using the following reaction mixture: 5X PrimeScript buffer, $2 \mu \mathrm{l}$; PrimeScript Enzyme mix, $0.5 \mu \mathrm{l}$; Oligo dT Primer, $0.5 \mu \mathrm{l}$; random 6 mers, $0.5 \mu \mathrm{l}$; total RNA, $500 \mu \mathrm{g}$; RNase-free $\mathrm{dH}_{2} \mathrm{O}, \leq 10 \mu \mathrm{l}$. RT conditions were as follows: $37^{\circ} \mathrm{C}, 15 \mathrm{~min} ; 85^{\circ} \mathrm{C}, 5 \mathrm{sec}$; and a final hold at $4^{\circ} \mathrm{C}$. The USP39 primers used were: forward 5'-CCGCTCGAGATG TCCGGCCGGTCTAAGC-3', and reverse 5'-CGGAATTCG AGCCCCCTGCTGGTTGGTTTC-3', which contains restriction endonuclease XhoI and EcoRI sites. The endonuclease used was purchased from Tiangen Biotech Co., Ltd. PCR cycling conditions were as follows: $95^{\circ} \mathrm{C}$ for $5 \mathrm{~min}, 95^{\circ} \mathrm{C}$ for $30 \mathrm{sec}, 55^{\circ} \mathrm{C}$ for $30 \mathrm{sec}, 72^{\circ} \mathrm{C}$ for $2 \mathrm{~min}(30$ cycles $), 72^{\circ} \mathrm{C}$ for $10 \mathrm{~min}$, and a final hold at $4^{\circ} \mathrm{C}$. The products of PCR reaction and the empty vectors pEGFP-N2 (BD Biosciences, Franklin Lakes, NJ, USA) were joined together and transformed into DH5 $\alpha$ E. coli (GeneChem Co., Ltd.), and the recombinant plasmids were subsequently extracted using a plasmid extraction kit (Tiangen Biotech Co., Ltd.). Recombinant plasmids were transfected into cells using Lipofectamine 3000 (Invitrogen; Thermo Fisher Scientific, Inc.) following the manufacturer's protocol.

Cell growth. Cell growth was measured by multiparametric high content screening (HCS). SMMC-7721 cells infected with either NC lentivirus or USP39 siRNA lentivirus were seeded at 2,000 cells/well in 96-well plates and incubated for 5 days in complete medium at $37^{\circ} \mathrm{C}$. Plates were processed with the ArrayScan High Content Platform (cat. no. ASN00004F; Thermo Fisher Scientific, Inc.) and kept at $4^{\circ} \mathrm{C}$ for up to $24 \mathrm{~h}$ prior to daily analysis. The system identified stained cells and reported the intensity and distribution of fluorescence in each cell. In each well, $\geq 800$ cells were analyzed. Images and data were stored in a Microsoft SQL database. 
MTT assay. SMMC-7721 cells $\left(1 \times 10^{5}\right.$ in $0.2 \mathrm{ml} /$ well) were seeded in three replicate 96 -well plates and cultured in complete medium at $37^{\circ} \mathrm{C}$ for one to five days. A total of $100 \mu 1(5 \mathrm{mg} / \mathrm{ml})$ MTT solution was added to each well and incubated at $37^{\circ} \mathrm{C}$ for $4 \mathrm{~h}$. Supernatant was removed and $150 \mu 1$ dimethyl sulfoxide was added to each well. The plate was oscillated for $30 \mathrm{~min}$ at room temperature. Absorbance at $490 \mathrm{~nm}$ was measured via microplater reader (ELx800; BioTek Instruments, Inc., Winooski, VT, USA) and the values were determined following background subtraction. All MTT experiments were repeated a minimum of three times.

Tumor xenografts. A total of 20 female BALB/c nude mice aged 4-6 weeks old (weight, 20-22 g) were obtained from the Laboratory Animal Center of the Affiliated Drum Tower Hospital of Nanjing University Medical School (Nanjing, China) and maintained in standard pathogen-free conditions (temperature, $18-22^{\circ} \mathrm{C}$; humidity, 50-60\%; 12-h light/dark cycle). Mice were fed a diet that consisted of flour (40\%), maize meal (25\%), bran (20\%) and beans (15\%) supplemented with fish meal, egg, yeast, bone meal and cod-liver oil. Mice were provided with ad libitum access to food and water. A total of $2 \times 10^{6}$ tumor cells in $0.2 \mathrm{ml}$ serum-free RPMI-1640 medium were subcutaneously injected into the flank of each mouse. Some mice were injected with tumor cells containing USP39 shRNA, while the control group was injected with tumor cells containing non-silencing RNA. The experimental and control group each consisted of 10 mice and three mice within the control groups succumbed by day 8 . Tumor growth was maintained for 22 days and monitored by measuring the length (L) and width (W) of tumors using a caliper and tumor size was calculated by the formula $\mathrm{L} \mathrm{x} \mathrm{W}^{2} \mathrm{x}(\pi / 6)$. Mice were then sacrificed via cervical dislocation and tumors were isolated from the mice and embedded in paraffin with $4 \%$ paraformaldehyde fixative at room temperature. All experiments were approved by the Institutional Animal Care and Use Committee of Nanjing University (Nanjing, China).

RT-quantitative PCR (RT-qPCR). Total RNA was extracted from HCC cell lines using TRIzol (Invitrogen; Thermo Scientific, Inc.) following the manufacturer's protocol. RT-qPCR was performed as reported previously (23). A total of $1 \mu \mathrm{g}$ RNA was transcribed using random primers and Primescript reverse transcriptase (Takara Biotechnology Co., Ltd., Dalian, China). qPCR for USP39 and GAPDH was conducted using a SYBR green qPCR kit (Takara Biotechnology Co., Ltd.) on a fluorescent temperature cycler (Mx3000P Real Time PCR system; Agilent Technologies, Inc., Santa Clara, CA, USA). The following primers were used to detect the expression of USP39: Forward, 5'-CCAGCG ATGGCAACTAC-3' and reverse, 5'-ACCACAACGGAA ACACG-3'; GAPDH: Forward, 5'-TGACTTCAACAGCGA CACCCA-3' and reverse, 5'-CACCCTGTTGCTGTAGCC AAA-3'). PCR was performed with the following parameters: Denaturation at $95^{\circ} \mathrm{C}$ for $5 \mathrm{~min}$, followed by 45 cycles at $95^{\circ} \mathrm{C}$ for $15 \mathrm{sec}$ and $60^{\circ} \mathrm{C}$ for $1 \mathrm{~min}$. Using GAPDH as a loading control, relative gene expression was determined using the ${ }^{\Delta 4} C_{q}$ method (23). Gene expression was analyzed with MxPro version 1.0 software (Agilent Technologies, Inc.). Experiments were repeated a minimum of three times.
Flow cytometry. Cells transfected with lentivirus were harvested, washed twice with cold phosphate-buffered saline (PBS), fixed at $4^{\circ} \mathrm{C}$ with cold $70 \%$ ethanol overnight and resuspended in PBS. The suspension was filtrated through a 400-mesh membrane. The cells were stained with propidium iodide or Annexin V-APC (eBioscience, Inc., San Diego, CA, USA) and analyzed using a BD FACSCalibur Flow Cytometer with Kaluza Analysis software (version 1.3; BD Biosciences). Experiments were repeated a minimum of three times.

Cell colony formation. SMMC-7721 cells were resuspended and seeded into 6-well plates at a final concentration of 200 cells/well and cultured at $37^{\circ} \mathrm{C}$ for 14 days. The medium was replaced every 3 to 4 days. Following the incubation period, the cells were washed with PBS twice and fixed with $4 \%$ paraformaldehyde at room temperature for $1 \mathrm{~h}$. The cells were washed with PBS twice, stained with Giemsa (Sigma-Aldrich; Merck Millipore, Darmstadt, Germany) for $10 \mathrm{~min}$ and washed with $\mathrm{ddH}_{2} \mathrm{O}$ three times. The plates were photographed with a digital camera. Experiments were repeated a minimum of three times.

Immunohistochemistry. Following deparaffinization of tumor tissue, antigen retrieval was performed in citrate buffer (Nanjing KeyGen Biotech Co., Ltd., Nanjing, China). Non-specific binding was blocked with $0.3 \% \mathrm{H}_{2} \mathrm{O}_{2}$ (Nanjing KeyGen Biotech Co., Ltd.) for $10 \mathrm{~min}$, and with $10 \%$ goat serum (Nanjing KeyGen Biotech Co., Ltd.) at room temperature for $30 \mathrm{~min}$. Samples were then incubated at $4^{\circ} \mathrm{C}$ overnight with anti-PCNA (dilution, 1:500) and anti-USP39 antibodies (dilution, 1:200). Samples were washed in buffer and incubated with the goat anti-mouse IgG-HRP secondary antibody (cat. no. KGAA37; dilution, 1:1,000; Nanjing KeyGen Biotech Co., Ltd.) for $45 \mathrm{~min}$, followed by the diaminobenzidine-peroxidase reaction and counterstaining with hematoxylin. Negative control sections were stained under identical conditions by omitting the primary antibody, and incubating with PBS. Stained sections were subsequently observed with light microscopy (Leica Microsystems GmbH) and evaluated using Image-Pro Plus 6.0 (Media Cybernetics, Inc., Rockville, MD, USA).

Western blotting. Total protein was extracted from tumor cell lines using radioimmunoprecipitation assay buffer (Nanjing KeyGen Biotech Co., Ltd.) containing fresh protease and phosphatase inhibitors. Protein concentration was determined using the BCA assay (Pierce; Thermo Fisher Scientific, Inc.). A total of $50 \mu \mathrm{g}$ protein per lane was separated on $10 \%$ SDS-PAGE and transferred onto a PVDF membrane. The membrane was blocked with $3 \%$ bovine serum albumin in $10 \mathrm{mM}$ Tris- $\mathrm{HCl}$ (pH 7.4) containing $0.05 \%$ Tween- 20 and incubated with primary antibodies for USP39, FoxM1, PLK1, cyclin $\mathrm{B} 1$ and CENPA at $4^{\circ} \mathrm{C}$ for $12 \mathrm{~h}$. Following 3 washes with Tris- $\mathrm{HCl}$ buffer, the membrane was incubated with a corresponding peroxidase-conjugated secondary antibody (cat no. sc-2005; dilution, 1:8,000; Santa Cruz Biotechnology, Inc.) and developed in Super-Signal West Pico Chemiluminescent Substrate (Pierce Protein Biology; Thermo Fisher Scientific, Inc.). The protein was visualized by autoradiography and quantified by densitometric analysis using a Versadoc Imaging 
A

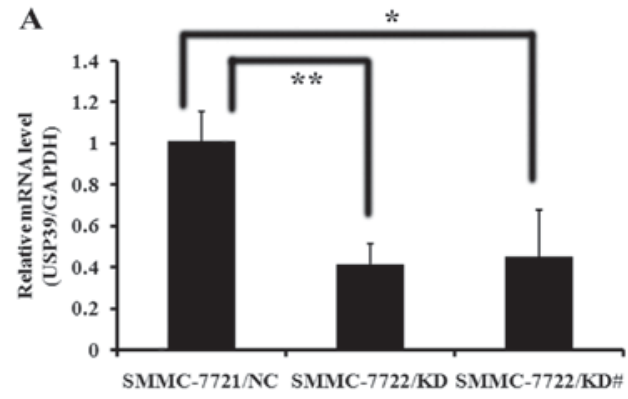

C

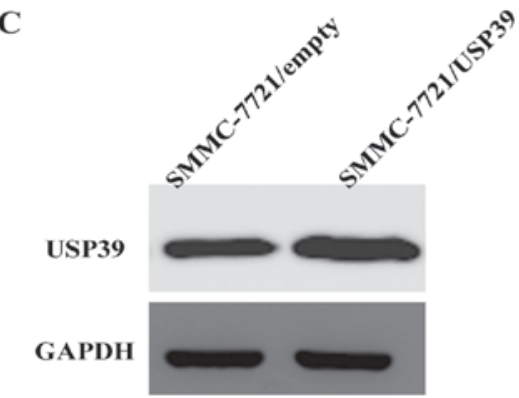

B

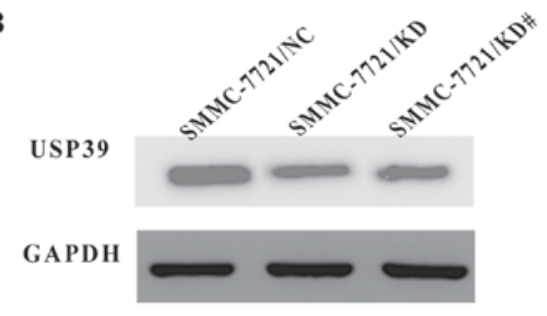

Figure 1. Efficiency of knockdown and overexpression of USP39 in SMMC-7721 cells. (A) The expression of USP39 mRNA relative to the expression of internal control protein GADPH in SMMC-7721/NC, SMMC-7721/KD and SMMC-7721/KD\# cells was examined with reverse transcription-quantitative polymerase chain reaction. (B) The expression of USP39 protein in SMMC-7721/NC, SMMC-7721/KD and SMMC-7721/KD\# cells was examined by western blot analysis using GAPDH as an internal control. (C) Overexpression efficiency of USP39 in SMMC-7721 cells was detected by western blot analysis using GAPDH as an internal control. ${ }^{\mathrm{P}}<0.05,{ }^{* *} \mathrm{P}<0.01$. USP39, ubiquitin specific peptidase 39.

system model 3000 (Bio-Rad Laboratories, Inc., Hercules, CA, USA). Experiments were repeated a minimum of three times.

Statistical analyses. The data shown are presented as the mean \pm standard deviation of three independent experiments. All statistical analyses were performed using the SPSS 11.0 software (SPSS Inc., Chicago, IL, USA). The xenografted tumor was analyzed with the paired $t$-test. $\mathrm{P}<0.05$ was considered to indicate a statistically significant difference.

\section{Results}

Downregulation and upregulation of USP39 in SMMC-7721 cells. To verify the roles of USP39 in tumorigenesis of HCC in vitro, USP39 expression was inhibited in SMMC-7721 cells by shRNA targeting of USP39. The expression of USP39 mRNA was significantly attenuated in SMMC-7721/KD $(\mathrm{P}<0.01)$ and SMMC-7721/KD\# $(\mathrm{P}<0.05)$ compared with SMMC-7721/NC (Fig. 1A). The expression of USP39 protein was also decreased in SMMC-7721/KD and SMMC-7721/KD\# cells compared with the control (Fig. 1B). However, USP39 protein expression was markedly increased in SMMC-7721/USP39 cells compared with SMMC-7721/vector cells at the protein level (Fig. 1C), indicating that SMMC-7721/USP39 cells successfully overexpress USP39.

USP39 knockdown inhibits growth of SMMC-7721 cells and USP39 upregulation enhances growth of SMMC-7721 cells. To explore the function of USP39 on cell growth, SMMC-7721 cells transfected with either USP39-siRNA lentivirus or NC lentivirus were monitored by cellomics assay. SMMC-7721 cells containing USP39-siRNA and NC were seeded in 96-well plates and cell growth was assayed every day for 5 days (Fig. 2A). The results demonstrated that cell growth was inhibited when USP39 was knocked down (P<0.01; Fig. 2B). MTT assays also indicated that growth of SMMC-7721 cells was significantly increased after USP39 was overexpressed $(\mathrm{P}<0.01$; Fig. $2 \mathrm{C})$. These results indicated that USP39 knockdown suppressed the growth of HCC cells and USP39 overexpression promoted HCC cell proliferation.

USP39 induces G2/M arrest and suppresses cell colony formation. It has been demonstrated that USP39 regulates the cell cycle checkpoint (20-22). Therefore, changes in the cell cycle were examined using flow cytometry and the colony formation capacity of SMMC-7721 was determined. It was observed that SMMC-7721 cells with USP39 knockdown exhibited a reduced cell population in the G0/G1 stage and a greater proportion of cells in the $\mathrm{G} 2 / \mathrm{M}$ stage $(\mathrm{P}<0.01$; Fig. 3A). SMMC-7721/KD cells and SMMC-7721/KD\# cells also showed an increased rate of apoptosis compared with control cells $(\mathrm{P}<0.01$; Fig. 3B). Furthermore, the colony formation capacity of SMMC-7721 cells after USP39 knockdown was investigated. HCC cells were grown for 14 days to form colonies. The number of SMMC-7721 colonies was significantly decreased following USP39 knockdown compared with control cells $(\mathrm{P}<0.05$; Fig. 4).

Effect of USP39 on xenograft tumor growth. To investigate the effects of USP39 on xenograft tumor growth, SMMC-7721 cells containing USP39 shRNA or non-silencing RNA were injected into nude mice. USP39 knockdown significantly decreased tumor growth in SMMC-7721 cells $(\mathrm{P}<0.05$; Fig. 5A and $\mathrm{B})$. In addition, cell proliferation in SMMC-7721/KD tumors was decreased as demonstrated by anti-PCNA staining (Fig. 5C). 
A
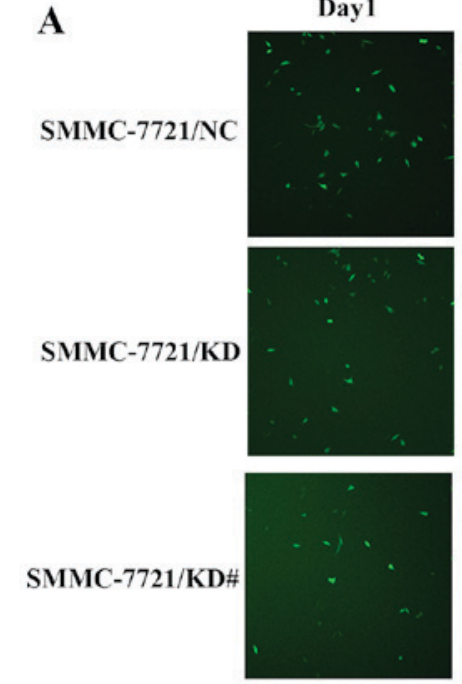

B

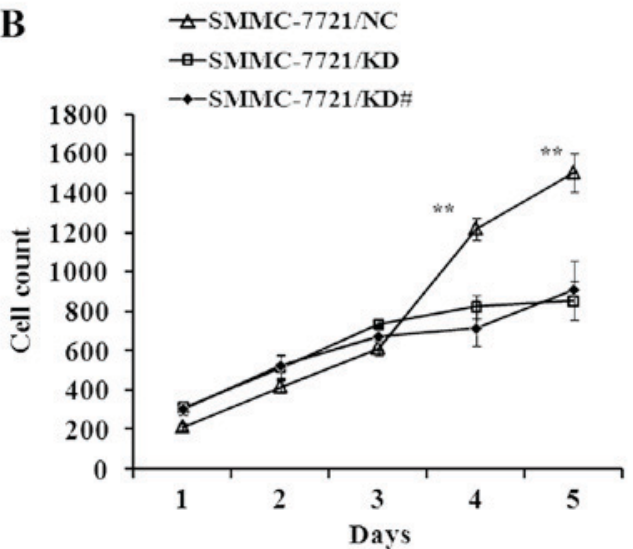

Day 2
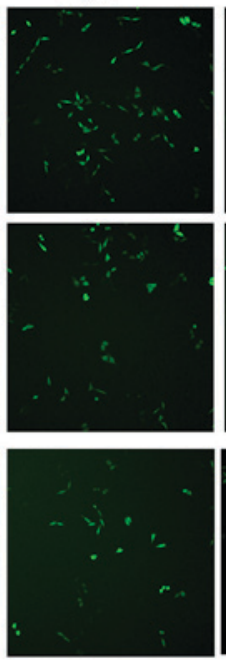

C
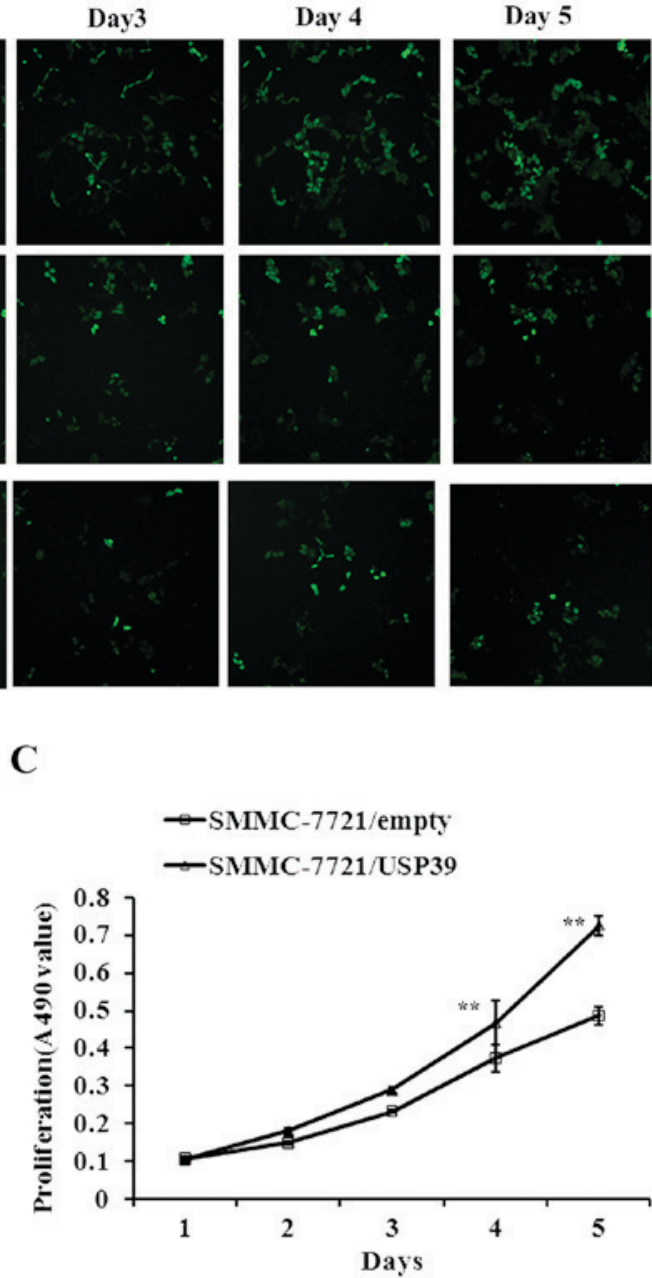

Figure 2. Effect of downregulating and upregulating USP39 on SMMC-7721 cell growth. (A) USP39-siRNA targeted cells and negative control cells were observed by cellomics for 5 days. (B) Cell count curve of USP39-siRNA targeted cells and negative control cells. Cell proliferation was significantly inhibited in SMMC-7721/KD cells and SMMC-7721/KD\# cells compared with negative control cells. ${ }^{* *} \mathrm{P}<0.01$ in SMMC-7721/KD and SMMC-7721/KD\# cells vs. negative controls. (C) MTT assay to test cell proliferation in SMMC-7721/empty and SMMC-7721/USP39 cells revealed overexpression of USP39 significantly enhanced the growth of tumor cells in SMMC-7721 cells. ${ }^{* * *} \mathrm{P}<0.01$ in SMMC-7721/USP39 cells vs. SMCC-7721/empty cells. siRNA, small interfering RNA; USP39, ubiquitin specific peptidase 39

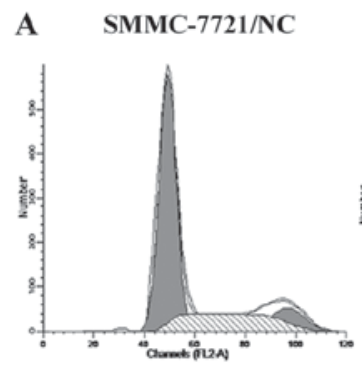

B SMMC-7721/NC

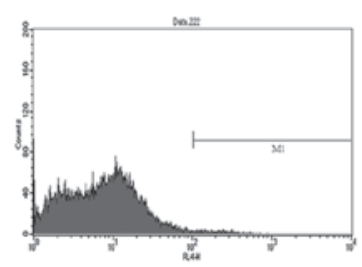

SMMC-7721/KD

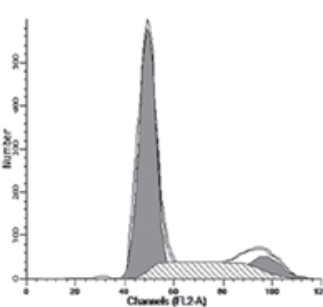

SMMC-7721/KD

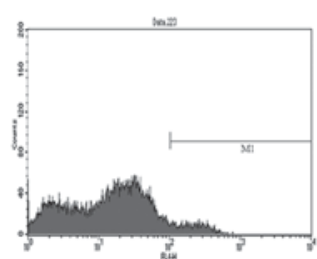

SMMC-7721/KD\#

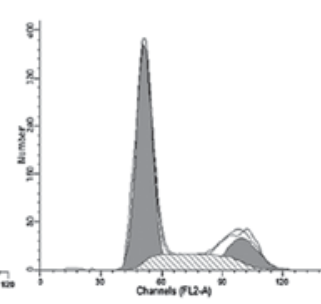

SMMC-7721/KD\#

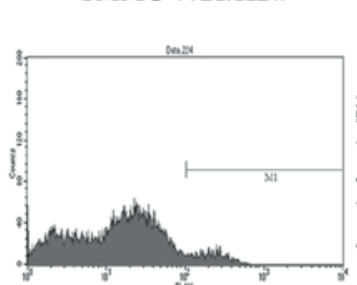

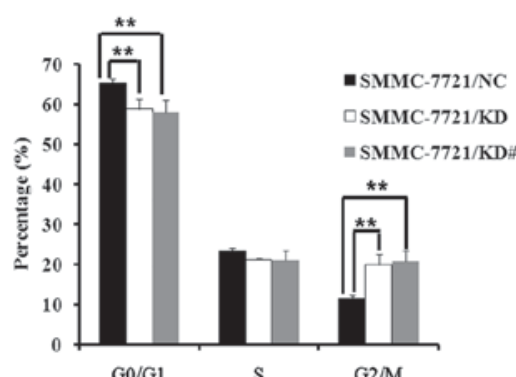

G0/G1

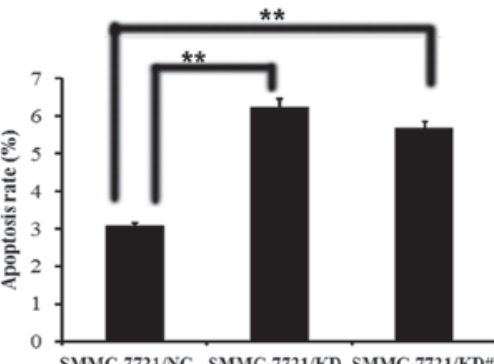

Figure 3. USP39 knockdown inhibits the growth of SMMC-7721 cells in vitro. Flow cytometry analysis was used to examine cell cycling in SMMC-7721 cells for 5 days following virus infection. (A) The percentage of cells in the G2/M phase was significantly increased and (B) the apoptosis rate was significantly enhanced following USP39 knockdown. ${ }^{* *} \mathrm{P}<0.01$. USP39, ubiquitin specific peptidase 39. 
A

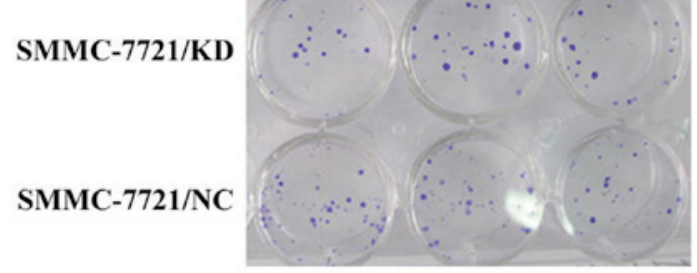

C

SMMC-7721/KD\#

SMMC-7721/NC

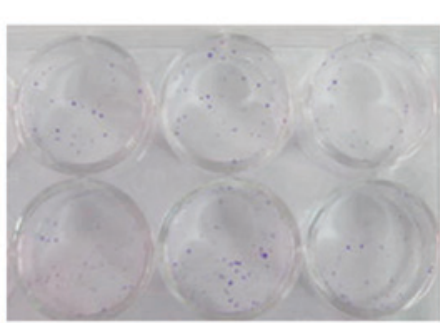

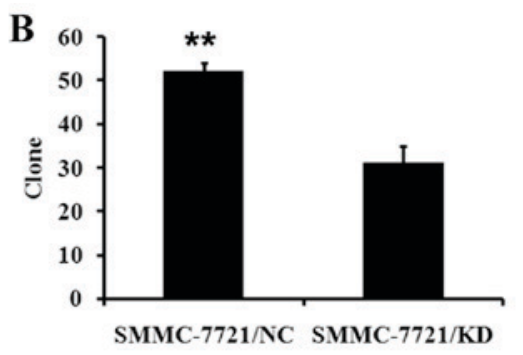

D

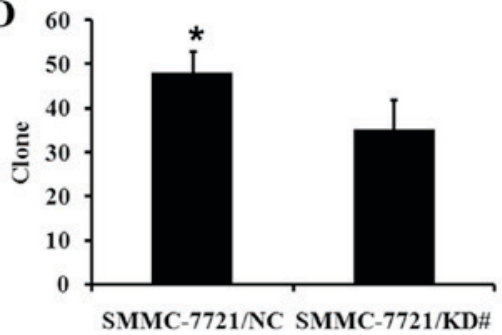

Figure 4. Colony formation following downregulation of USP39 in SMMC-7721 cells. (A and B) Cell colony formation in SMMC-7721/KD cells was significantly decreased compared with control cells. ${ }^{* *} \mathrm{P}<0.01$ in SMMC-7721/KD cells vs. control. (C and D) Cell colony formation in SMMC-7721/KD\# cells was significantly decreased compared with control cells. " $\mathrm{P}<0.05$ in SMMC-7721/KD\# cells vs. control.

A

SMMC-7721/NC

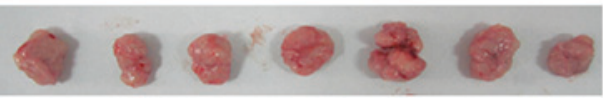

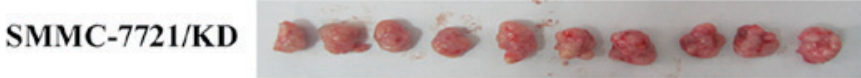

B
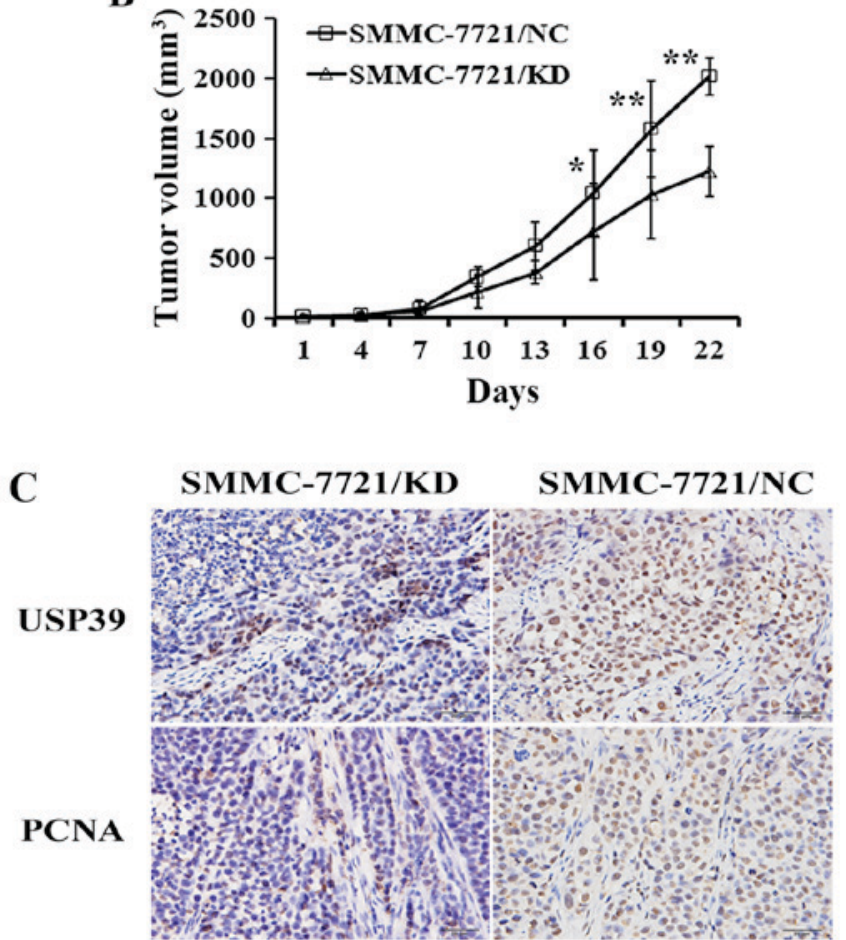

Figure 5. USP39 knockdown inhibits growth of SMMC-7721 cells in vivo. $\mathrm{n}=10$ (3 mice within the control groups succumbed by day 8); (A) USP39 knockdown in SMMC-7721 cells decreased xenografted tumor growth in nude mice. (B) Tumor growth was significantly inhibited. (C) USP39 knockdown in SMMC-7721 cells (top panels) decreased cell proliferation (lower panels) in xenografted tumors. ${ }^{*}<0.05,{ }^{* *} \mathrm{P}<0.01$ vs. control. USP39, ubiquitin specific peptidase 39 ; $\mathrm{PCNA}$, proliferating cell nuclear antigen.

These results indicate that USP39 contributes to HCC tumor growth in vivo.
USP39-induced G2/M arrest depends on FoxM1. To further investigate the underlying molecular mechanisms by which 

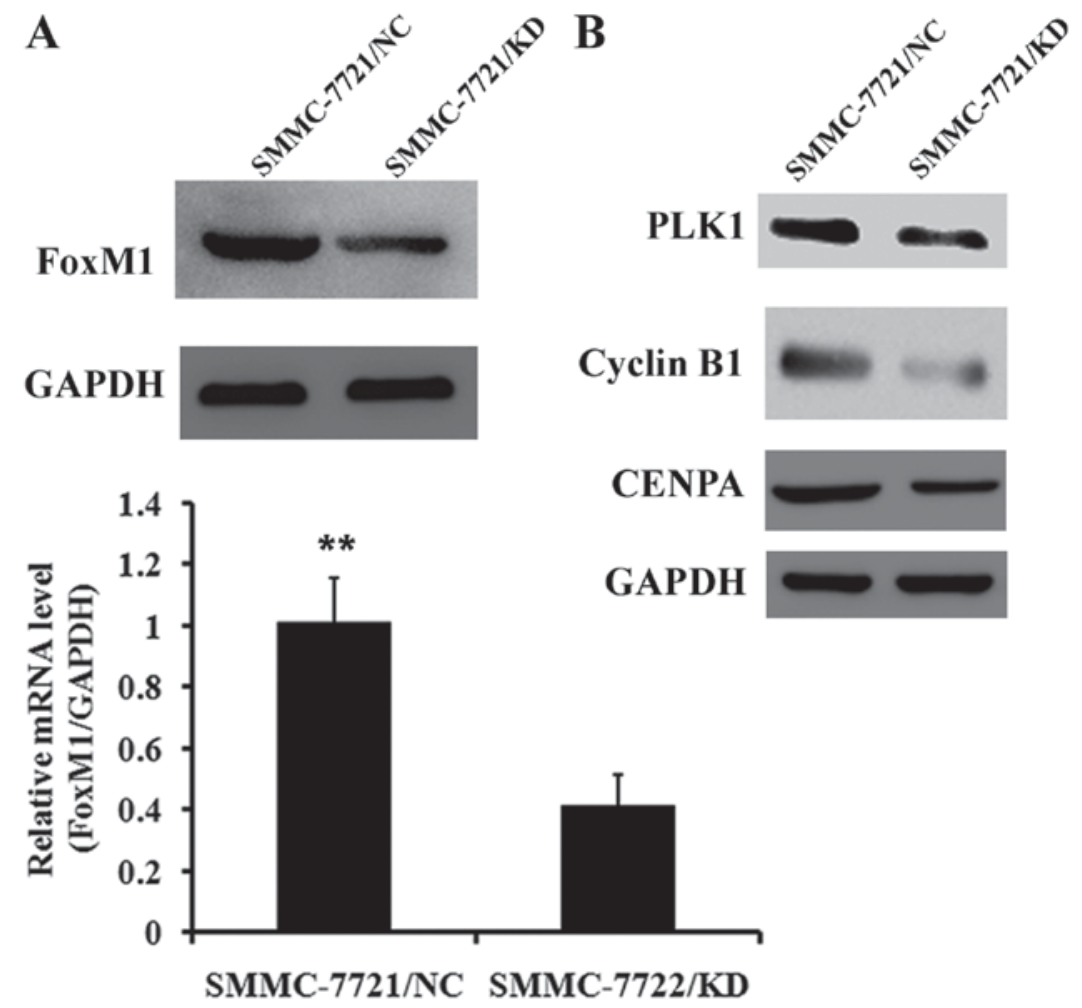

Figure 6. Mechanisms for USP39 regulating tumor growth. Western blot analysis was conducted to detect the expression of cell cycle checkpoint proteins FoxM1, PLK1, cyclin B1 and CENPA. (A) FoxM1 was significantly decreased following USP39 knockdown in SMMC-7721 cells at the mRNA level and markedly decreased at the protein level. (B) Expression of PLK1, cyclin B1 and CENPA were downregulated following USP39 knockdown in SMMC-7721 cells. Fox M1, Forkhead Box M1; PLK1, polo-like kinase 1; CENPA, centromere protein A; USP39, ubiquitin specific peptidase 39. ${ }^{* *}$ P<0.01 vs. SMMC-7721/KD.

USP39 regulates HCC cell proliferation, the expression of important cell cycle proteins was examined. Since USP39 is important in pre-mRNA splicing and FoxM1 is regulated by mRNA splicing, it was hypothesized that FoxM1 was involved in pre-mRNA splicing induced by USP39. The expression of FoxM1 was determined at the mRNA and protein levels (Fig. 6A). The expression of FoxM1 mRNA was significantly decreased following USP39 knockdown $(\mathrm{P}<0.01)$. The expression of genes downstream of FoxM1 including PLK1, cyclin B1 and CENPA was also decreased following USP39 knockdown (Fig. 6B).

\section{Discussion}

HCC is one of the most prevalent tumors worldwide and has a high mortality rate, particularly in China (24). Gene therapy has attracted increasing attention in recent years. Meanwhile, RNA interference (RNAi) has also developed rapidly over the past few decades. During RNAi, double stranded siRNA degrades target mRNA, subsequently inhibiting protein synthesis of the associated target gene (25). Identification of novel target genes for further research is in progress. In the current study, it was demonstrated that USP39 knockdown by siRNA causes cell cycle arrest in the G2/M phase of HCC cells.

Stable lentiviral vectors were constructed, containing two siRNA sequences for the knockdown of endogenous USP39 and a construct containing USP39 cDNA was created to overexpress USP39. It was demonstrated that USP39 knockdown significantly blocked the growth and colony formation of SMMC-7721 cells. By contrast, USP39 overexpression promoted the growth of SMMC-7721 cells. Moreover, USP39 knockdown resulted in $\mathrm{G} 2 / \mathrm{M}$ arrest and induced apoptosis in SMMC-7721 cells. These results imply that USP39 knockdown inhibited HCC growth, possibly by inducing G2/M arrest. Furthermore, tumor growth was decreased in SMMC-7721/KD engrafted mice compared with control mice. Thus, USP39 knockdown inhibited the growth of HCC cells in vitro and in vivo.

Cell-cycle checkpoints control the proper timing of cell-cycle events by enforcing dependency of later events on the completion of earlier events in the cycle (26). Consequently, checkpoint blockage results in cell-cycle arrest and alters cell proliferation. It has been indicated that the downregulation of USP39 may inactivate the G0/G1 arrest in the zebrafish in vivo by splicing the rb1 protein, which serves an important role in the transition from G0 to G1 phase (21). A similar effect has been observed in human breast cancer cells (22). The results of the present study showed that USP39 knockdown induced G2/M arrest in SMMC-7721 cells. Levels of FoxM1 were decreased following USP39 knockdown in SMMC-7721 cells and a similar downregulatory effect on some of the downstream factors of FoxM1 including PLK1, CENPA and cyclin B1, was observed. It is well known that these genes are all involved in the G2/M transition (27). Therefore, USP39 knockdown may induce G2/M arrest, leading to suppression of HCC growth, possibly through the modulation of FoxM1 mRNA splicing.

In conclusion, using in vitro and in vivo approaches, the present study provides evidence that USP39 knockdown 
inhibits tumor growth in human $\mathrm{HCC}$ by inducing $\mathrm{G} 2 / \mathrm{M}$ arrest. This may be partly due to blockage of FoxM1 splicing, suggesting that USP39 may be considered as a promising molecular target to treat HCC.

\section{Acknowledgements}

The present study was supported by the National Natural Science Foundation of China (grant no. 31300103), the Clinical Medical Center for Hepatobiliary Disease of Jiangsu Province (grant no. ZX201105) and the Clinical Medical Center for Digestive Disease of Jiangsu Province (grant no. BL2012001).

\section{References}

1. Wang G, Chen JH, Qiang Y, Wang DZ and Chen Z: Decreased STAT4 indicates poor prognosis and enhanced cell proliferation in hepatocellular carcinoma. World J Gastroenterol 21: 3983-3993, 2015.

2. Villanueva A, Minguez B, Forner A, Reig M and Llovet JM: Hepatocellular carcinoma: Novel molecular approaches for diagnosis, prognosis and therapy. Annu Rev Med 61: 317-328, 2010.

3. Huynh H: Molecularly targeted therapy in hepatocelluar carcinoma. Biochem Pharmacol 80: 550-560, 2010.

4. Fisher D, Krasinska L, Coudreuse D and Novák B: Phosphorylation network dynamics in the control of cell cycle transitions. J Cell Sci 125: 4703-4711, 2012.

5. Fu Z, Malureanu L, Huang J, Wang W, Li H, van Deursen JM, Tindall DJ and Chen J: Plk1-dependent phosphorylation of FoxM1 regulates a transcriptional programme required for mitotic progression. Nat Cell Biol 10: 1076-1082, 2008.

6. Korver W, Roose J and Clevers H: The winged-helix transcription factor Trident is expressed in cycling cells. Nucleic Acids Res 25: 1715-1719, 1997.

7. Ye H, Kelly TF, Samadani U, Lim L, Rubio S, Overdier DG, Roebuck KA and Costa RH: Hepatocyte nuclear factor 3/fork head homolog 11 is expressed in proliferating epithelial and mesenchymal cells of embryonic and adult tissues. Mol Cell Biol 17: 1626-1641, 1997.

8. Yao KM, Sha M, Lu Z and Wong GG: Molecular analysis of a novel winged helix protein, WIN. Expression pattern, DNA binding property, and alternative splicing within the DNA binding domain. J Biol Chem 272: 19827-19836, 1997.

9. Yoshida Y, Wang IC, Yoder HM, Davidson NO and Costa RH: The forkhead box M1 transcription factor contributes to the development and growth of mouse colorectal cancer. Gastroenterology 132: 1420-1431, 2007.

10. Nagata A, Igarashi M, Jinno S, Suto K and Okayama H: An additional homolog of the fission yeast $c d c 25^{+}$gene occurs in humans and is highly expressed in some cancer cells. New Biol 3: 959-968, 1991 .
11. Galaktionov K and Beach D: Specific activation of cdc25 tyrosine phosphatases by B-type cyclins: Evidence for multiple roles of mitotic cyclins. Cell 67: 1181-1194, 1991.

12. Molinari M, Mercurio C, Dominguez J, Goubin F and Draetta GF: Human Cdc25A inactivation in response to $S$ phase inhibition and its role in preventing premature mitosis. EMBO Rep 1: 71-79, 2000.

13. Karlsson C, Katich S, Hagting A, Hoffmann I and Pines J: $\mathrm{Cdc} 25 \mathrm{~B}$ and $\mathrm{Cdc} 25 \mathrm{C}$ differ markedly in their properties as initiators of mitosis. J Cell Biol 146: 573-584, 1999.

14. De Souza CP, Ellem KA and Gabrielli BG: Centrosomal and cytoplasmic Cdc2/cyclin B1 activation precedes nuclear mitotic events. Exp Cell Res 257: 11-21, 2000.

15. Hoffmann I, Clarke PR, Marcote MJ, Karsenti E and Draetta G: Phosphorylation and activation of human cdc25-C by cde2-cyclin $\mathrm{B}$ and its involvement in the self-amplification of MPF at mitosis. EMBO J 12: 53-63, 1993.

16. Perdiguero E and Nebreda AR: Regulation of cdc 25 c activity during the meiotic G2/M transtition. Cell Cycle 3: 733-737, 2004.

17. Schmitt A and Nebreda AR: Signalling pathways in oocyte meiotic maturation. J Cell Sci 115: 2457-2459, 2002.

18. Qian Y, Erikson E, Taieb FE and Maller JL: The polo-like kinase p1x1 is required for activation of the phosphatase $\mathrm{Cdc} 25 \mathrm{C}$ and cyclin B-Cdc2 in Xenopus oocytes. Mol Biol Cell 12: 1791-1799, 2001.

19. Reyes-Turcu FE, Ventii KH and Wilkinson KD: Regulation and cellular roles of ubiquitin-specific deubiquitinating enzymes. Annu Rev Biochem 78: 363-397, 2009.

20. Van Leuken RJ, Luna-Vargas MP, Sixma TK, Wolthuis RM and Medema RH: Usp39 is essential for mitotic spindle checkpoint integrity and controls mRNA-levels of aurora B. Cell Cycle 7: 2710-2719, 2008.

21. Ríos Y, Melmed S, Lin S and Liu NA: Zebrafish usp39 mutation leads to rb1 mRNA splicing defect and pituitary lineage expansion. PLoS Genet 7: e1001271, 2011.

22. Wang H, Ji X, Liu X, Yao R, Chi J, Liu S, Wang Y, Cao W and Zhou Q: Lentivirus-mediated inhibition of USP39 suppresses the growth of breast cancer cells in vitro. Oncol Rep 30: 2871-2877, 2013.

23. Yuan X, Sun X, Shi X, Jiang C, Yu D, Zhang W, Guan W, Zhou J, Wu Y, Qiu Y and Ding Y: USP39 promotes the growth of human hepatocellular carcinoma in vitro and in vivo. Oncol Rep 34: 823-832, 2015.

24. Sun XT, Yuan XW, Zhu HT, Deng ZM, Yu DC, Zhou X and Ding YT: Endothelial precursor cells promote angiogenesis in hepatocellular carcinoma. World J Gastroenterol 18: 4925-4933, 2012.

25. Lindon $\mathrm{C}$ and Pines J: Ordered proteolysis in anaphase inactivates Plk1 to contribute to proper mitotic exit in human cells. J Cell Biol 164: 233-241, 2004.

26. Makarova OV, Makarov EM and Luhrmann R: The 65 and $110 \mathrm{kDa}$ SR-related proteins of the U4/U6.U5 tri-snRNP are essential for the assembly of mature spliceosomes. EMBO J 20: 2553-2563, 2001.

27. Hanahan D and Weinberg RA: Hallmarks of cancer: The next generation. Cell 144: 646-674, 2011. 